Anales de Geografía de la Universidad Complutense ISSN: 0211-9803

http://dx.doi.org/10.5209/AGUC.72979

\title{
Evaluación del índice de vulnerabilidad costera en la Manga del Mar Menor (Murcia, España) ${ }^{1}$
}

\author{
Daniel Ibarra Marinas²; Gustavo A. Ballesteros Pelegrín³; Jorge Sánchez Balibrea \\ Pedro García Moreno ${ }^{5}$; Francisco Belmonte Serrato ${ }^{6}$ \\ Recibido: 31 de marzo del 2020 / Enviado a evaluar: 20 de abril del 2020 / Aceptado: 15 de diciembre del 2020
}

Resumen. Uno de los efectos más visibles del Cambio Climático es la subida del nivel del mar por el derretimiento de las masas de hielo terrestre. Para calcular la vulnerabilidad costera de la Manga del Mar Menor por la subida del nivel del mar, se ha aplicado el Índice de Vulnerabilidad Costera utilizando las variables geomorfología, pendiente de la costa, cambios en el nivel del mar, erosión costera, rango mareal, oleaje y una variable ambiental. El resultado refleja que tramos de playas de arena y las estructuras artificiales presentan mayor vulnerabilidad a los riesgos climáticos que aquellos tramos rocosos, por lo que se considera necesario la adecuación de sistemas duna-playa con vegetación natural bien conservada que constituyan una barrera frente a la energía de los temporales.

Palabras clave: Cambio climático; duna-playa; resiliencia; variables.

\section{[en] Evaluation of the coastal vulnerability index in the Manga del Mar Menor (Murcia, Spain)}

\begin{abstract}
One of the most visible effects of Climate Change is the rise of sea levels by melting land ice masses. To calculate the coastal vulnerability of the Lower Sea Sleeve by sea level rise, the Coastal Vulnerability Index has been applied using geomorphology, coastal slope, sea level changes, coastal erosion, tidal range, swell and an environmental variable. The result reflects that stretches of sandy beaches and artificial structures are more vulnerable to climate risks than those rocky sections, so it is considered necessary to adapt dune-beach systems with natural vegetation well preserved that constitute a barrier to the energy of the storms.

Keywords: Climate change; dune-beach; resilience; Variables.

1 Este artículo se ha realizado gracias a la financiación de Fondos LIFE de la Unión Europea, a través del Proyecto LIFE17 NAT/ES/000184 Conservación de los hábitats y aves acuáticas en el LIC y ZEPA ES0000175 "Salinas y Arenales de San Pedro del Pinatar" (LIFE-SALINAS).

2 Departamento de Geografía. Universidad de Murcia. España.

E-mail: gabp1@um.es

3 Departamento de Geografía. Universidad de Murcia. España.

4 Asociación de Naturalistas del Sureste (ANSE).

5 Asociación de Naturalistas del Sureste (ANSE).

6 Departamento de Geografía. Universidad de Murcia. España.
\end{abstract}




\section{[fr] Évaluation de l'indice de vulnérabilité côtière à La Manga del Mar Menor (Murcie, Espagne)}

Résumé. L'un des effets les plus visibles du changement climatique est l'élévation du niveau de la mer due à la fonte des masses de glace terrestre. Pour calculer la vulnérabilité côtière de Manga del Mar Menor en raison de l'élévation du niveau de la mer, l'indice de vulnérabilité côtière a été appliqué en utilisant les variables de géomorphologie, la pente de la côte, les changements du niveau de la mer, l'érosion côtière, l'amplitude des marées, vagues et une variable environnementale. Le résultat reflète que les étendues de plages de sable et les structures artificielles sont plus vulnérables aux risques climatiques que ces étendues rocheuses, il est donc jugé nécessaire d'adapter les systèmes de dunes-plages avec une végétation naturelle bien préservée qui constitue une barrière contre la l'énergie des tempêtes.

Mots clés: Changement climatique; plage de dunes; résilience; les variables.

Cómo citar. Ibarra Marina, D., Ballesteros Pelegrín, G.A., Sánchez Balibrea, J., García Moreno, P. y Belmonte Serrato, F. (2020): Evaluación del índice de vulnerabilidad costera en la Manga del Mar Menor (Murcia, España). Anales de Geografía de la Universidad Complutense, 40(2), 373-392.

Sumario. 1. Introducción. 2. Área de estudio. 3. Metodología. 3.1. Índices de Vulnerabilidad Costera. 3.2. Variables empleadas. 4. Resultados. 5. Discusión. 6. Conclusiones. 7. Referencias.

\section{Introducción}

El cambio climático afecta de forma global a las áreas costeras de diferentes formas. El litoral es sensible al aumento del nivel del mar, a los cambios en la frecuencia e intensidad de las tormentas, los cambios en las precipitaciones y las temperaturas oceánicas más cálidas. Además, a escala mundial, las crecientes concentraciones atmosféricas de dióxido de carbono $\left(\mathrm{CO}_{2}\right)$ debido al aumento de la absorción de gas por el océano, hacen que el mar se vuelva más ácido, afectando a la cadena trófica (Rossoll et al., 2012). En la actualidad existe una relación directa entre la ocupación antropogénica y el desequilibrio natural del litoral (Moore y Griggs, 2002) que se ha convertido en un problema importante en el ámbito mundial.

Entre los efectos más visibles del Cambio Climático se encuentra la subida del nivel del mar por el derretimiento de las masas de hielo terrestre, principalmente las situadas en la Antártida y Groenlandia. Desde los años 90 el aumento del nivel del mar en la zona sur del Mediterráneo, estuvo en torno a los $2.5 \mathrm{~mm} /$ año (VargasYáñez et al., 2010), lo que provoca, en algunas playas, un retroceso significativo de la línea de costa.

Existen muchos índices de vulnerabilidad (Baccarin et al., 2017), que se crearon inicialmente para satisfacer las necesidades locales y posteriormente se adaptaron para otros contextos.

Una de las herramientas más importantes para evaluar los efectos del Cambio climático, utilizadas desde el primer Panel Internacional sobre Cambio Climático (IPCC) en 1990 son las relacionadas con la evaluación de la vulnerabilidad (IPCC, 1990), que puede definirse como la propensión de un área a ser afectada de forma 
negativa (IPCC, 2014) y que puede incluir tanto variables medioambientales como socioeconómicas (Baccarin, 2016). Uno de los problemas que se plantean en los estudios de vulnerabilidad es la escala. El hecho de aplicar índices a escala global, puede implicar cierto grado de simplificación (McLaughlin y Cooper, 2011).

La vulnerabilidad costera está relacionada con el grado en el que un determinado sector de costa se puede ver afectado por un evento específico (Adger, 2006). En este sentido, es una estimación semicuantitativa de distintas variables que permite identificar las zonas de mayor fragilidad del litoral (Rangel-Buitrago y Posada-Posada, 2013).

En los estudios de áreas costeras, este análisis constituye una de las herramientas más utilizadas a la hora de valorar los impactos (Gornitz et al., 1994; Shaw et al. 1998). El concepto de vulnerabilidad puede ser geofísico (Gornitz, 1991), social o socio-ambiental, y uno de los enfoques más comunes para abordar entre otras variables, el impacto producido por aumento del nivel del mar en diferentes escalas espaciales (Thieler y Hammar, Klose, 1999). Las áreas costeras bajas son severamente vulnerables al aumento del nivel del mar, por los de impactos ecológicos y sociales que puedan generar (Ballesteros, 2014; Nicholls y Cazenave, 2010; Wetzel et al., 2012), más aún en áreas costeras con alta densidad de infraestructuras y construcciones (Scott et al., 2012; Ojeda-Zujar et al., 2009; Hernández-Delgado, 2015) y en las que las dunas prácticamente han desaparecido. En este sentido, evaluar la vulnerabilidad permite la zonificación de las dunas costeras en unidades de manejo adecuadas (García-Mora et al., 2001).

El objetivo de este trabajo ha sido realizar una aproximación de la vulnerabilidad de La Manga del Mar Menor ante un escenario de Cambio Climático, utilizando las variables comunes en los estudios de Vulnerabilidad Costera.

\section{2. Área de estudio}

La Manga del Mar Menor se presenta como un cordón arenoso sobre un sustrato litológico discontinuo debido a la presencia de las golas (Figura 1). La sedimentación del sustrato arenoso se llevó a cabo durante el Cuaternario, apoyándose en los esquistos paleozoicos, rocas volcánicas y areniscas miocénicas (Lillo Carpio, 1978).

Hasta la formación de La Manga, el Mar Menor era una bahía en la que el desplazamiento de los sedimentos por parte de la dinámica litoral se vio reducido por la presencia de bajos rocosos, que surgieron tras una serie de erupciones volcánicas que formaron islas, las cuales fueron reteniendo las arenas procedentes de las ramblas litorales (Díaz del Río, 1990), principal fuente de sedimentos de las playas del sureste peninsular (Belmonte Serrato et al., 2013). Estos aportes finalmente formaron la barra. El transporte longitudinal y el transversal fue en ocasiones simultáneo, provocado por acción del oleaje, sobre una plataforma con una batimetría tendida. 
Figura 1. Localización de La Manga del Mar Menor.

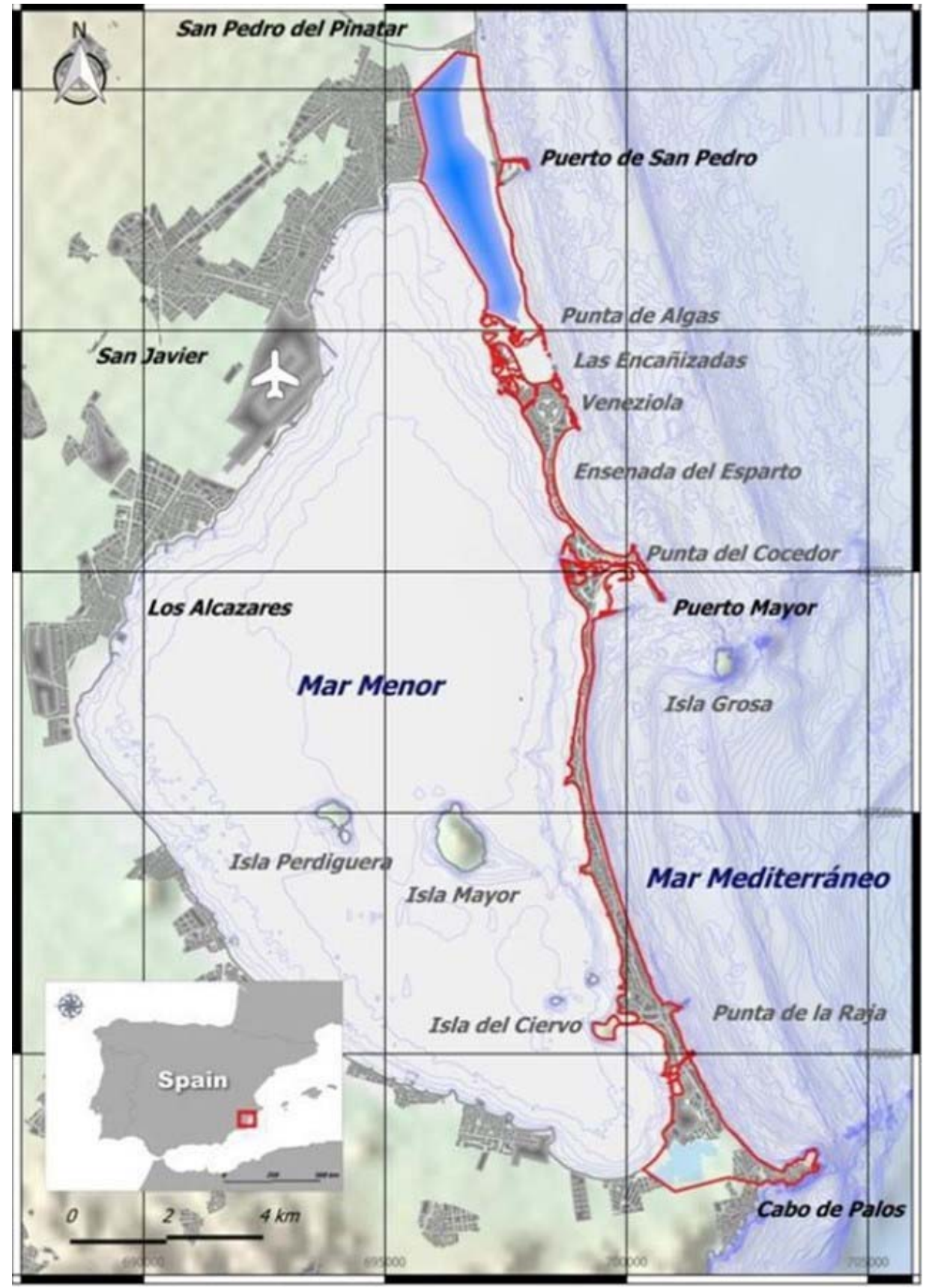

Fuente: Elaboración propia. 
A partir de los años 60 del s. XX, La Manga del Mar Menor sufrió un importante proceso de urbanización que selló prácticamente la totalidad de los sistemas dunares que alimentaban las playas y que ha supuesto una pérdida de biodiversidad, incluyendo la desaparición de especies como el enebro de las dunas (Juniperus macrocarpa).

\section{Metodología}

\section{1. Índices de Vulnerabilidad Costera}

Los Indices de Vulnerabilidad Costera (CVI) proporcionan una base numérica para clasificar secciones de la costa en términos de su potencial de cambio como resultado de varios factores, como la geomorfología, la geología, el aumento del nivel del mar y el oleaje. La información permite identificar sectores de la costa donde el riesgo puede ser relativamente alto y donde se deben desarrollar estrategias de gestión apropiadas (Appeaning, 2013). A pesar de que una gran mayoría de las evaluaciones de vulnerabilidad costera en todo el mundo se realizan a escala global o escala ciudad, existen estudios con escalas inferiores a escala de pequeña ciudad (Kantamaneni et al., 2017).

El CVI se construye a partir de un sistema de ponderación empleado variables permite su integración en una ecuación que combinando las variables de riesgo relativo, permite crear un solo indicador. la primera fase implica la creación de una base de datos de variables geológicas y ambientales (Gornitz y White, 1992). Las variables incluidas en esta base de datos son geología, geomorfología, elevación, tasas relativas de aumento del nivel del mar, tasas de recesión en la costa, rango de mareas y altura media de las olas. La segunda fase del estudio implica la evaluación de los impactos potenciales en la línea costera y en la vegetación dunar.

$$
C V I=\sqrt{\frac{a \cdot b \cdot c \cdot d \cdot e \cdot f \cdot g}{7}}
$$

Donde a representa la variable geomorfológica, b la pendiente, $\mathrm{c}$ los cambios en el nivel del mar por efecto del Cambio Climático, $\mathrm{d}$ la erosión costera, e el rango mareal y $\mathrm{f}$ el oleaje. En este trabajo se ha incluido una variable de tipo ambiental (g). La cuantificación de las variables se basa en la definición de puntajes semicuantitativos de acuerdo con una escala de 1-5 (Hammer-Klose y Thieler, 2001), donde 1 indica una contribución baja a la vulnerabilidad costera de una variable clave específica y 5 indica una contribución alta. 


\subsection{Variables empleadas}

Las distintas variables que se integran en el índice han sido calculadas en 52 celdas costeras de $500 \mathrm{~m}$, situadas a lo largo de la costa de La Manga del Mar Menor, desde el límite de la Región de Murcia con la Comunidad Valenciana hasta Cabo de Palos. Estas unidades han sido digitalizadas mediante el Sistema de Información Geográfica de código abierto QGIS.

a) Geomorfología. La variable de geomorfología expresa la erosionabilidad relativa de diferentes tipos de formas de relieve. Para clasificar esta variable se han digitalizado datos de tipología costera y que ha generado un modelo digital del terreno para las alturas.

b) Pendiente de la costa. La pendiente de la zona costera se calculó a partir de las elevaciones topográficas, mediante un modelo digital de elevaciones y batimétricas, a partir de transectos sobre un shapefile de batimetría de $1 \mathrm{~m}$ de resolución. Esta variable se extiende desde $10 \mathrm{Km}$ mar adentro y, debido a la escasa anchura de la restinga, incluye la totalidad de la restinga. La pendiente regional permite evaluar no solo el riesgo relativo de inundación, sino también la posible rapidez del retroceso costero, ya que las regiones costeras de baja pendiente deberían retirarse más rápido (Pilkey y Davis, 1987).

c) Cambios en el nivel del mar. La variable relativa del cambio del nivel del mar se ha obtenido a partir de los datos del mareógrafo de Valencia en mm/año. La estimación se ha calculado a partir de la regionalización de los datos locales a partir de la relación de mareógrafos de Church y White (2011) y utilizando el escenario RCP8.5 (Riahi et al., 2007). Aproximadamente, cada milímetro que sube el nivel del mar, supone una pérdida de 10 centímetros de costa (Simeoni, 2009), aunque las playas de menor pendiente tenderán a una mayor erosión.

d) Erosión costera. La erosión costera es uno de los principales problemas del litoral que se ha acrecentado por la acción del hombre. Para su cálculo se ha digitalizado la línea de costa a partir de las imágenes aéreas de 1956 y 2016. Este proceso presenta una gran complejidad y requiere el desarrollo de una técnica sólida, especialmente en las playas arenosas debido a su variabilidad natural (Pajak y Leatherman, 2002). Para el cálculo de los procesos de erosión/acreción de La Manga del Mar Menor se ha utilizado como indicador el "límite superior instantáneo" (Dolan et al., 1980; Overton et al., 1999; Ibarra et al., 2015) que es la diferencia de coloración que marca el límite húmedo/seco producido por el flujo de agua. Posteriormente se ha empleado el plugin Station Lines del software QGIS, con el fin de obtener las tasas de erosión.

e) Rango mareal. El rango de mareas está relacionado con riesgos de inundación tanto permanentes como episódicos (Thieler y Hammar-Klose, 2000). El rango de mareas es la diferencia vertical entre la pleamar y la bajamar, las playas del Mediterráneo son de naturaleza micromareal (Vidal et al., 1995), esto permite la acumulación de bermas vegetales (Roig i Munar y Martín Prieto, 2005) que contribuyen al mantenimiento de la línea de costa (Ibarra, 2016). Los datos se han obtenido del mareógrafo del Puerto de Cartagena, sistema NIVMAR. 
f) Oleaje. El oleaje es el principal agente modelador del litoral. Los datos de oleaje se han obtenido a partir del software Sistema de Modelado Costero (SMC) desarrollado por el Instituto Hidraúlico de Cantabria.

Existen numerosos trabajos que emplean las variables anteriormente mencionadas (Tabla 1), siguiendo la metodología de Gornitz (1991). Otros autores adoptan modificaciones dependiendo del objetivo del estudio (Kokot et al., 2004; Codignotto, 2005; Ojeda-Zujar et al., 2009; Fernández et al., 2017). En este caso, se ha añadido una variable ambiental, de tal forma que el índice trata de analizar la vulnerabilidad de la flora dunar de La Manga del Mar Menor respecto a las características de la costa. Existen también variaciones a la hora del cálculo de las variables, dependiendo de las fuentes de datos disponibles.

Tabla 1. Variables consideradas para el cálculo del Índice de Vulnerabilidad Costera (CVI) y su rango de valores modificado de Gornitz (1991).

\begin{tabular}{|c|c|c|c|c|c|}
\hline & Muy bajo & Bajo & Moderado & Alto & Muy alto \\
\hline Variable & 1 & 2 & 3 & 4 & 5 \\
\hline Geomorfología & $\begin{array}{l}\text { - Costas } \\
\text { rocosas y } \\
\text { acantiladas } \\
\text { - Fiordos }\end{array}$ & $\begin{array}{l}\text { - Acantilados } \\
\text { medios } \\
\text { - Costa } \\
\text { accidentada }\end{array}$ & $\begin{array}{l}\text { - Acantilados } \\
\text { bajos } \\
\text { - Deriva glaciar } \\
\text { - Llanura aluvial }\end{array}$ & $\begin{array}{l}\text { - Lagoons } \\
\text { - Estuarios } \\
\text { - Playas de } \\
\text { adoquines }\end{array}$ & $\begin{array}{c}\text { - Playas de } \\
\text { arena de barrera } \\
\text { - Salinas } \\
\text { - Deltas } \\
\text { - Arrecifes de } \\
\text { coral } \\
\text { - Manglares } \\
\text { - Marismas }\end{array}$ \\
\hline $\begin{array}{c}\text { Pendiente de la } \\
\text { costa }(\%)\end{array}$ & $>0,2$ & $0,2-0,7$ & $0,7-0,4$ & $0,4-0,025$ & $<0,025$ \\
\hline $\begin{array}{l}\text { Cambios en el } \\
\text { nivel del mar } \\
\text { (mm/año) }\end{array}$ & $>1,8$ & $1,8-2,5$ & $2,5-2,95$ & $2,95-3,16$ & $>3,16$ \\
\hline $\begin{array}{c}\text { Erosión/acreción } \\
\text { costera } \\
(\mathrm{mm} / \mathrm{año})\end{array}$ & $>-2,0$ & $1,0-2,0$ & Estable & $1,1--2,0$ & $>3,16$ \\
\hline $\begin{array}{c}\text { Rango mareal } \\
(\mathrm{m})\end{array}$ & $>1,0$ & $1,0-1,9$ & $2,0-4,0$ & $4,1-6,0$ & $>2,0$ \\
\hline Oleaje (m) & $>0,55$ & $0,55-0,85$ & $0,85-1,05$ & $1,05-1,25$ & $>6,0$ \\
\hline
\end{tabular}

Fuente: Elaboración propia.

g) Variable ambiental. El deterioro de la vegetación está íntimamente relacionado con la degradación de los sistemas duna-playa que dan protección a la costa, la desaparición de plantones afecta al desarrollo de las dunas embrionarias o primarias (Roig i Munar, 2004), debido a la compactación del suelo que provocan una descompensación de los balances sedimentarios del sistema (Bird, 1996), evitando el equilibrio de los sistemas. Sin embargo, en este caso se ha analizado precisamente la vulnerabilidad de hábitats prioritarios y la flora dunar. Para ello se ha integrado la información espacial de hábitats prioritarios y el catalogo de vegetación dunar elaborado por la Asociación de Naturalistas del Sureste (ANSE). 
Los valores utilizados han sido los siguientes: 1) Sin vegetación/presencia de especies exóticas; 2) presencia de especies no protegidas; 3) Presencia de especies de interés; 4) Presencia de especies vulnerables; 5) Hábitats prioritarios/presencia de especies en peligro.

\section{Resultados}

a) Geomorfología. La mayor parte de La Manga del Mar Menor tiene su origen en el aporte sedimentario procedente de la deriva litoral, por lo que la mayor parte de la costa es arenosa, con playas que poseen una pendiente reducida (Figura 2). Solamente en el sector meridional (Cabo de Palos) existen acantilados bajos. En el sector norte, las salinas de San Pedro del Pinatar están separadas de la costa por sistemas dunaplaya.

Figura 2. Clasificación de la variable "Geomorfología" a lo largo de la costa de La Manga del Mar Menor.

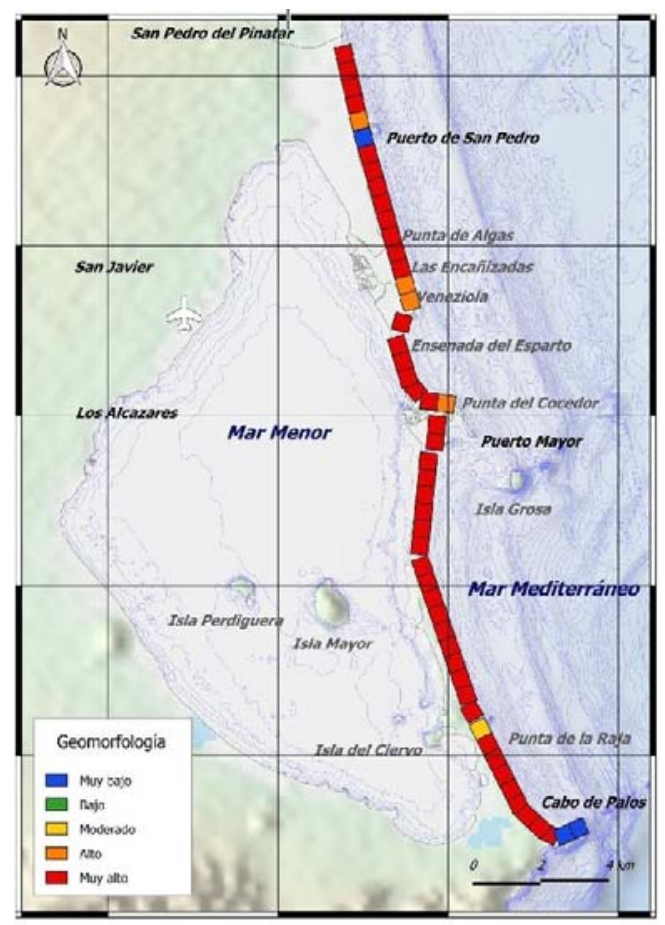

Fuente: Elaboración propia.

b) Pendiente de la costa. Debido a la propia génesis, no existen grandes relieves en la restinga (Figura 3). Por otro lado, la batimetría posee una pendiente escasa. 
Figura 3. Clasificación variable "Pendiente" a lo largo de la costa de La Manga del Mar Menor.

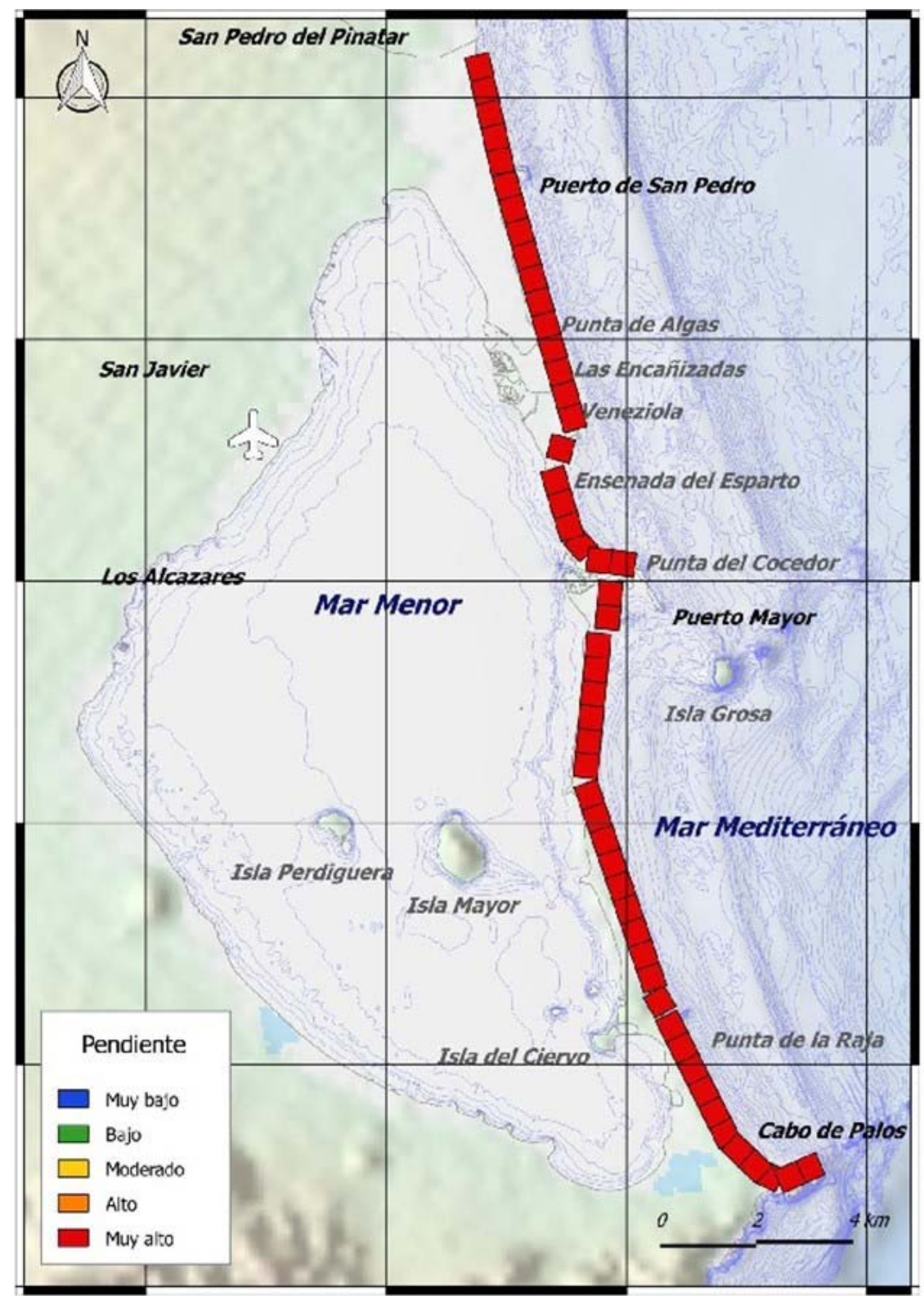

Fuente: Elaboración propia. 
c) Cambios en el nivel del mar. Los resultados del modelo de variaciones en el nivel del mar por efecto de Cambio Climático, muestran una subida de 1,4 m para el periodo 2080-2100 en el escenario RCP8.5, lo que sugiere una vulnerabilidad entre baja y muy baja (Figura 4). Variable que se debe tener en cuenta por las características de la costa.

Figura 4. Clasificación de la variable "Nivel del mar" a lo largo de la costa de La Manga del Mar Menor.

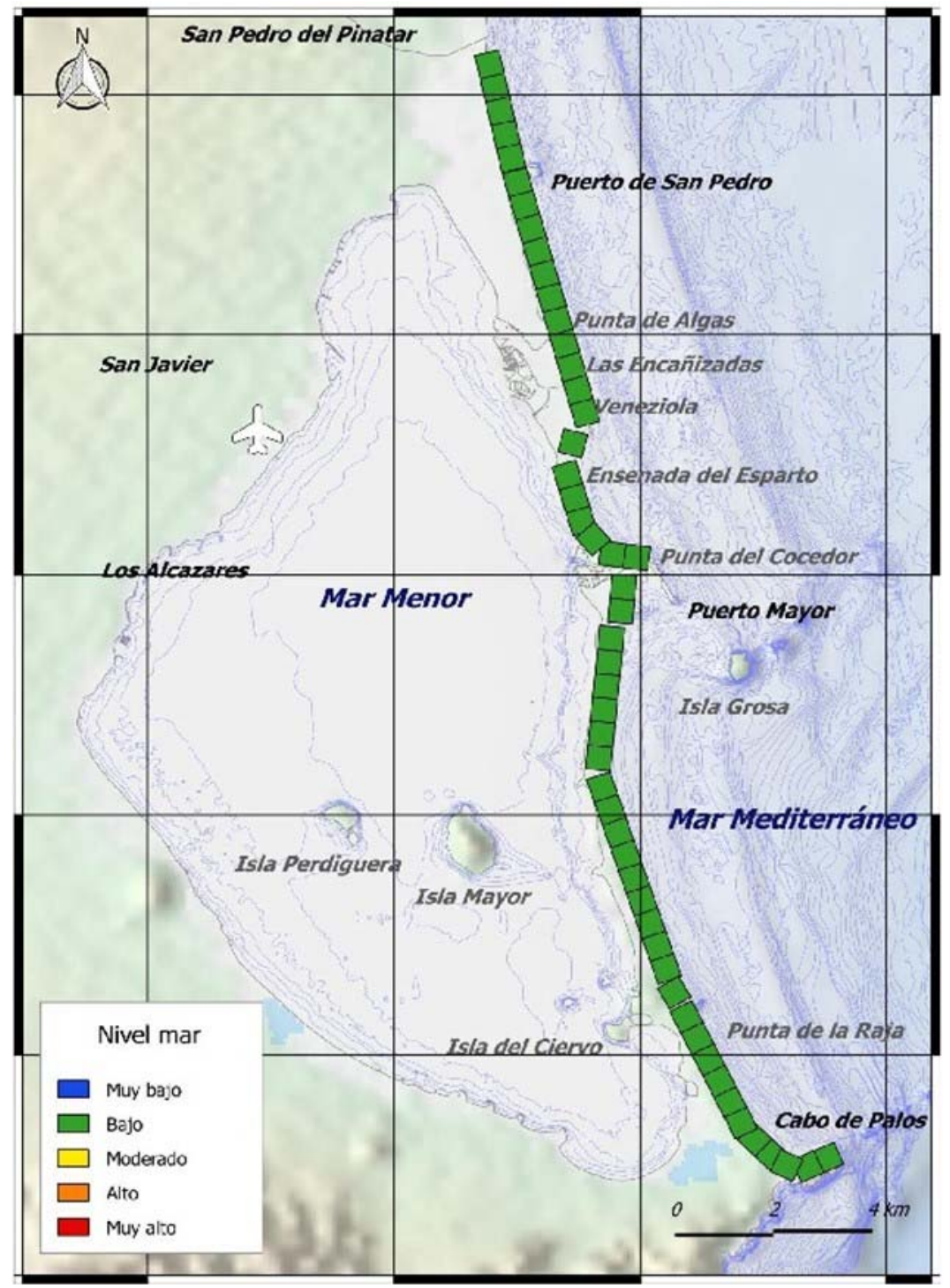

Fuente: Elaboración propia. 
d) Erosión costera. La ampliación del Puerto de San Pedro del Pinatar en el norte se hace notar en los sectores situados a barlomar, que poseen las mayores tasas de erosión de la Región de Murcia. En el resto de los sectores se encuentran en situación erosiva o estable, salvo las situadas en el sector norte y en el tramo situado al sur del Parque Regional de las Salinas y Arenales de San Pedro del Pinatar, junto a la gola de las Encañizadas, con una acreción moderada. Sin embargo, las celdas no muestran la realidad de los problemas erosivos en áreas determinadas (Figura 5).

Figura 5. Clasificación de la variable "Erosión costera" a lo largo de la costa de La Manga del M. Menor.

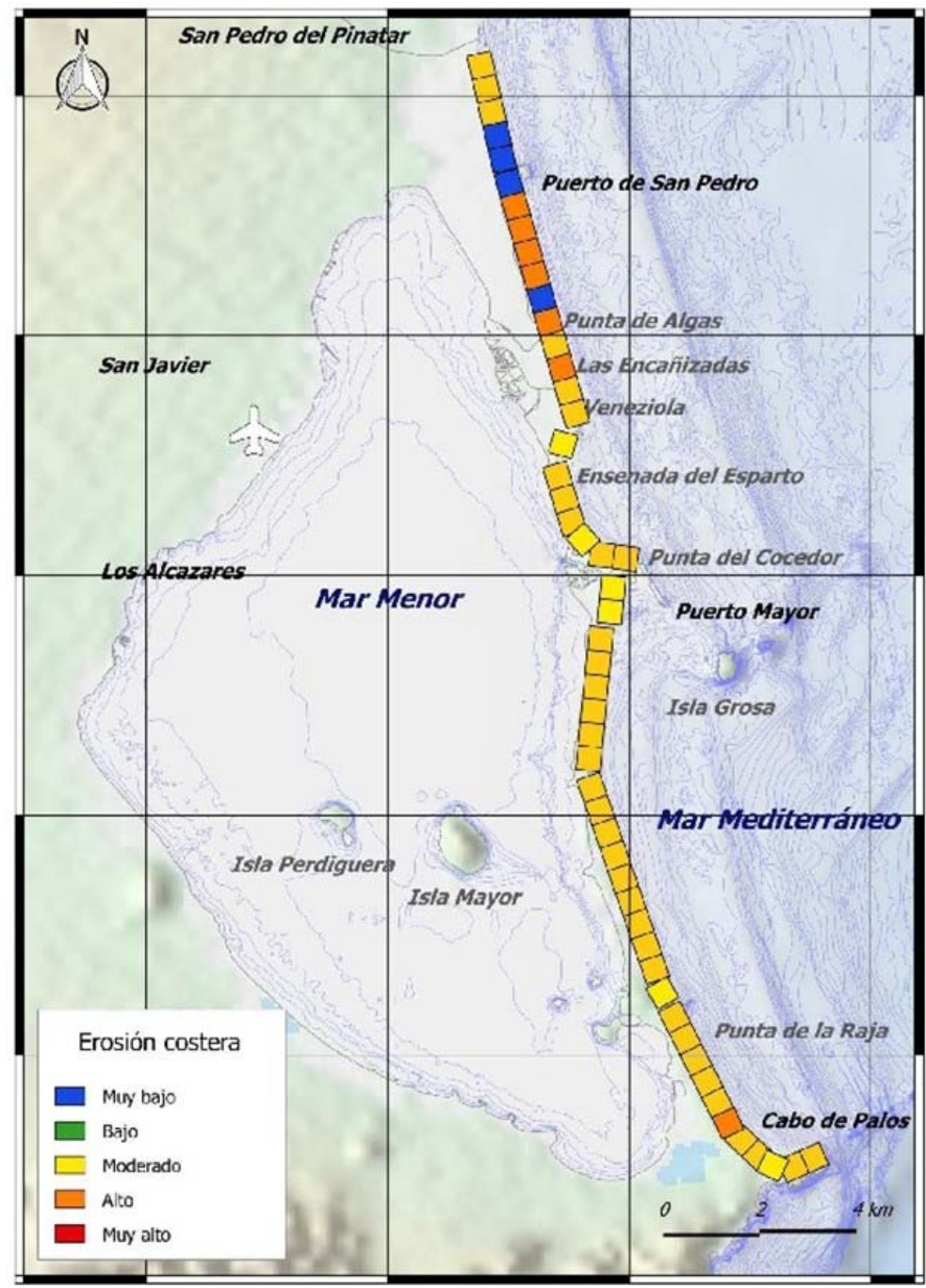

Fuente: Elaboración propia. 
e) Rango mareal. El Mediterráneo posee un carácter micromareal, por lo que es muy bajo (Figura 6).

Figura 6. Clasificación de la variable "Rango mareal" a lo largo de la costa de La Manga del Mar Menor.

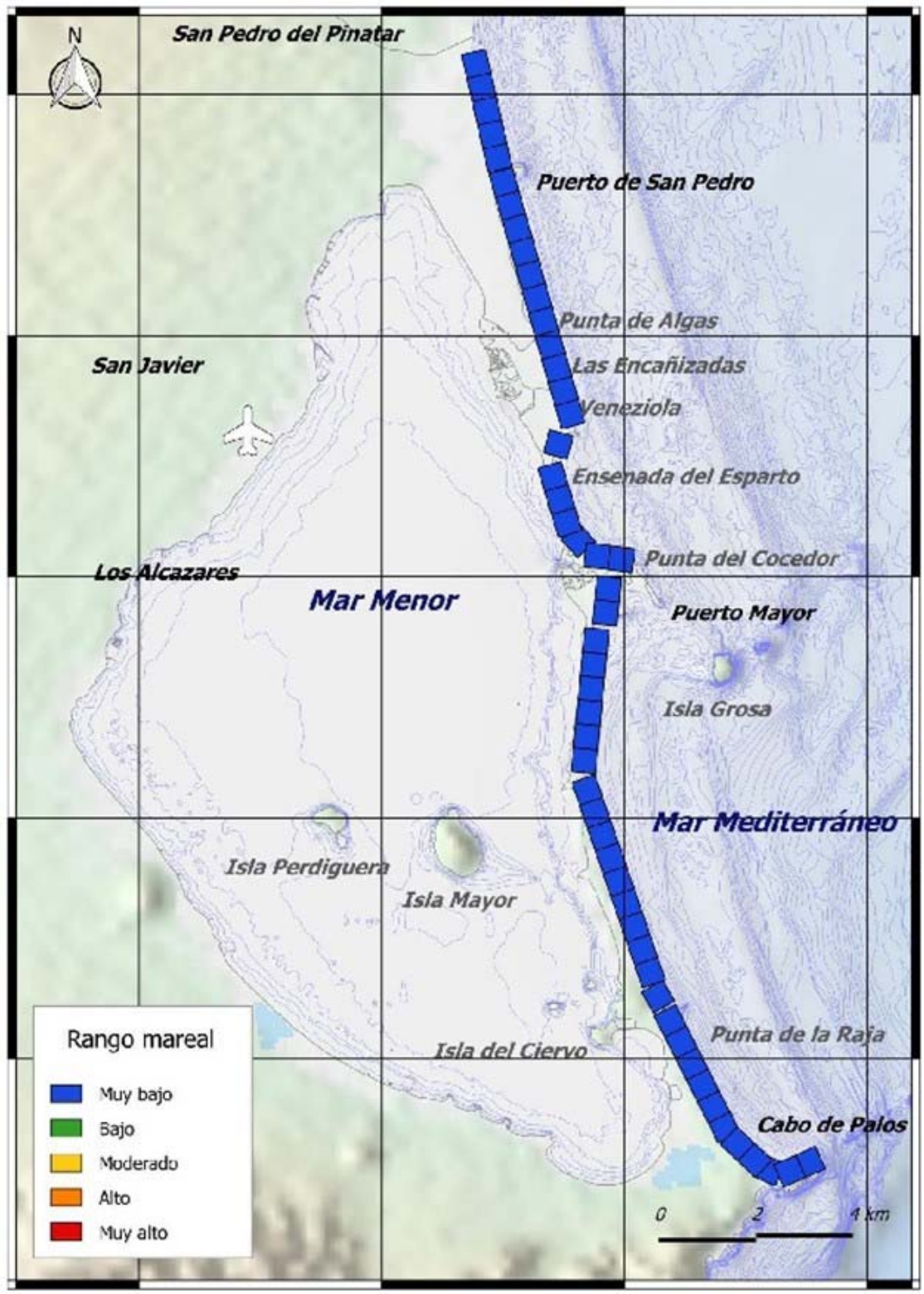

Fuente: Elaboración propia. 
f) Oleaje. Los datos obtenidos por medio del Sistema de Modelado Costero, presentan una altura de ola significante de $1,1 \mathrm{~m}$ en todos los tramos del estudio, por lo que se puede considerar alto a efectos de vulnerabilidad (Figura 7).

Figura 7. Clasificación de la variable "Oleaje" a lo largo de la costa de La Manga del Mar Menor.

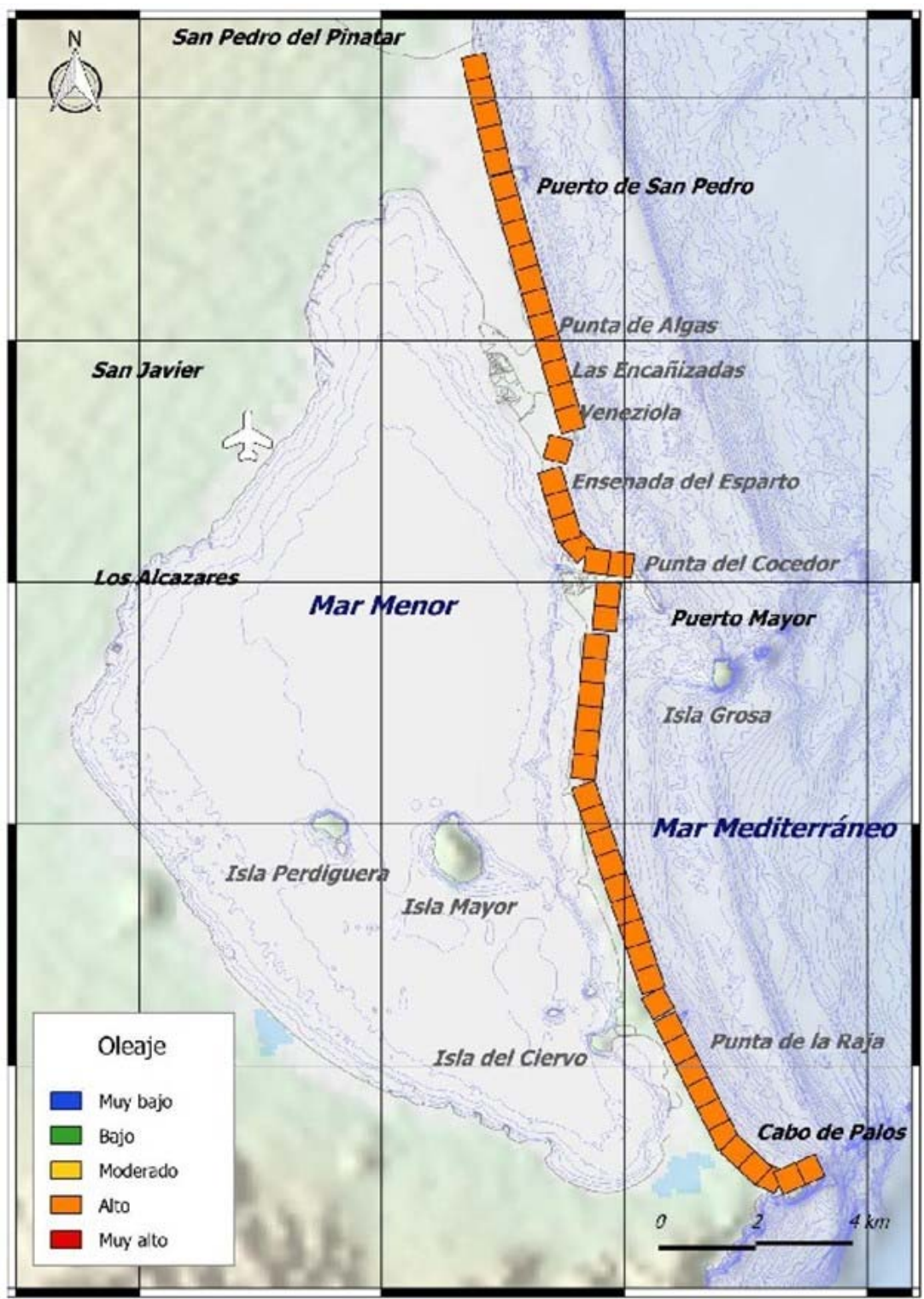

Fuente: Elaboración propia. 
g) Variable ambiental: presencia de flora dunar. Las zonas con mayor vulnerabilidad se sitúan en el Parque Regional de las Salinas y Arenales de San Pedro del Pinatar, en el tramo de Veneziola a Punta del Cocedor, al Sur de Puerto Mayor y al sur de Punta de la Raya. La presencia de flora dunar coincide en todos los casos con la presencia de dunas (Figura 8).

Figura 8. Clasificación de la vulnerabilidad de la vegetación dunar.

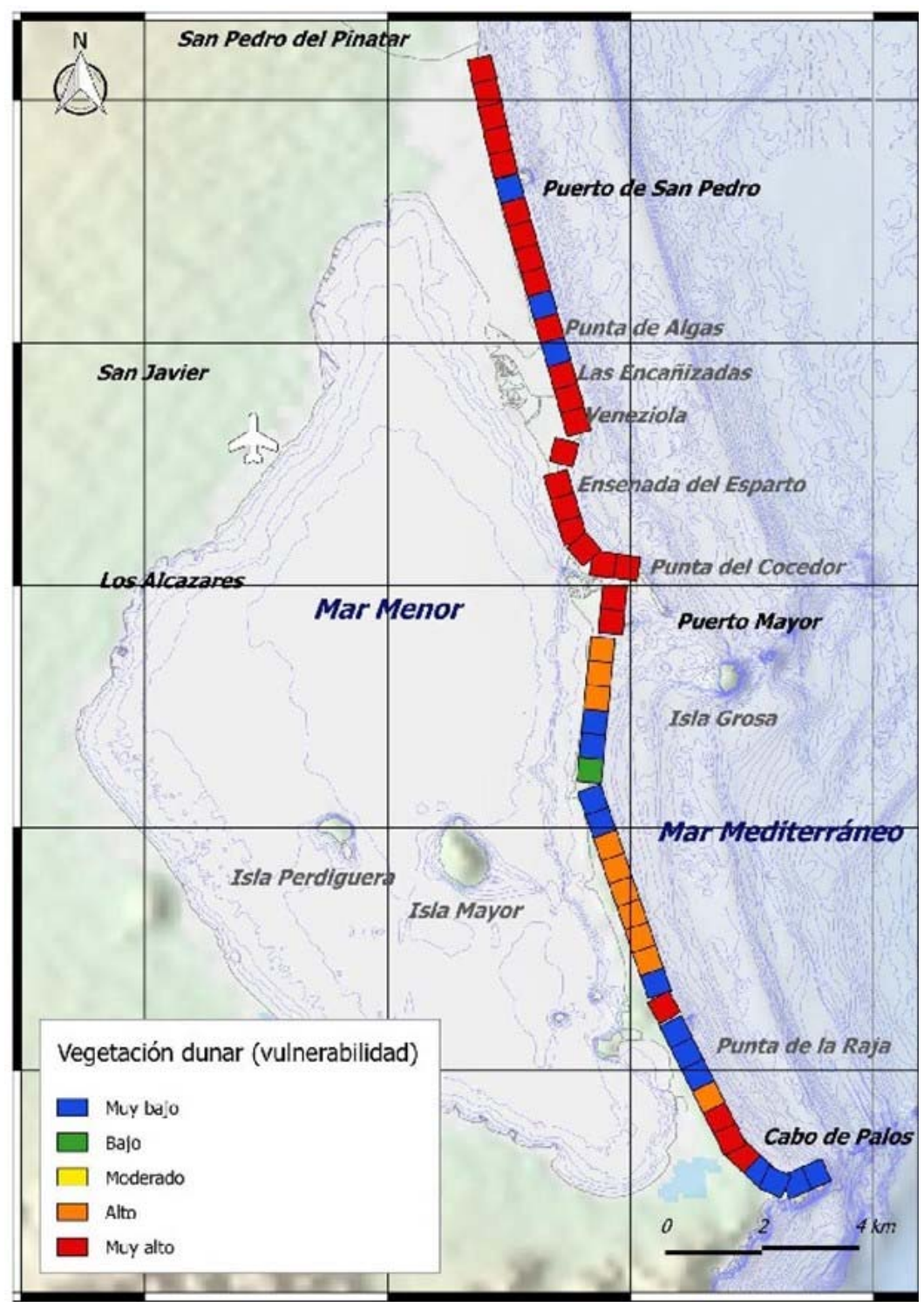

Fuente: Elaboración propia. 
h) Índice de Vulnerabilidad Ambiental. Como resultado final se ha obtenido el CVI adimensional para cada sector de costa. Las zonas costeras con mayor vulnerabilidad ambiental, se sitúan en el Parque Regional y las zonas dunares. Las zonas con menor vulnerabilidad ambiental se sitúan en los puertos y en la zona de Cabo de Palos. La media es 16,36 y la mediana 18,52. Los datos estadísticos obtenidos muestran un valor mínimo de 3,38 y un máximo de 23,90. La cartografía (Figuras 3-9) muestran los resultados de las variables y del CVI (Figura 9), mientras que los datos numéricos están representados en la Tabla 2.

Figura 9. Índice de Vulnerabilidad Costera y Ambiental aplicado a La Manga del Mar Menor.

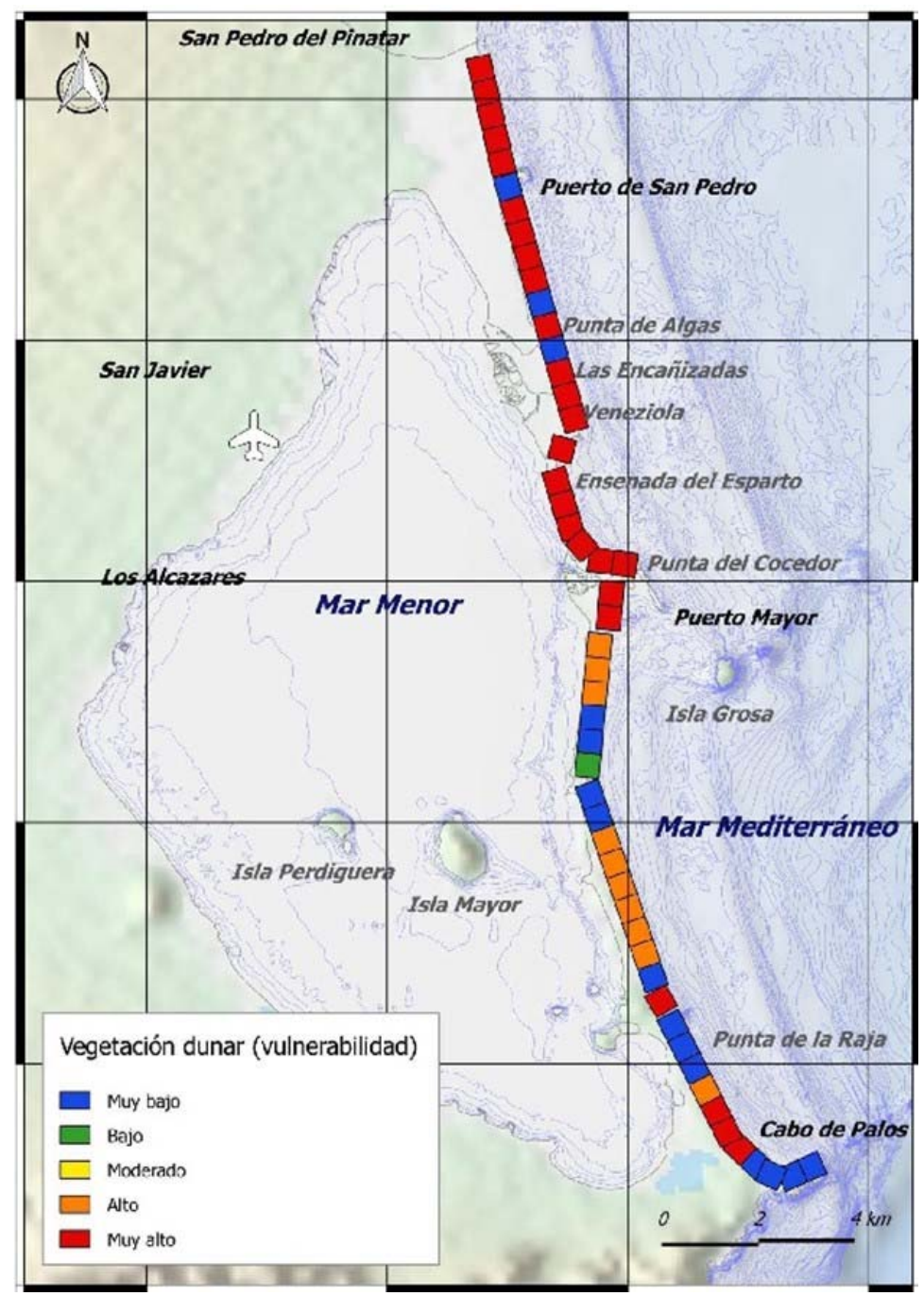

Fuente: Elaboración propia. 
Tabla 2. Valores de CVI para La Manga del Mar Menor.

\begin{tabular}{|c|c|c|c|c|c|c|c|c|}
\hline Celda & Geomorfología & Pendientes & Nivel del mar & Erosión & Mareas & Oleaje & Vul. vegetal & ICV \\
\hline 1 & 5 & 5 & 2 & 3 & 1 & 4 & 5 & 20,7 \\
\hline 2 & 5 & 5 & 2 & 3 & 1 & 4 & 5 & 20,7 \\
\hline 3 & 5 & 5 & 2 & 3 & 1 & 4 & 5 & 20,7 \\
\hline 4 & 5 & 5 & 2 & 2 & 1 & 4 & 5 & 16,9 \\
\hline 5 & 4 & 5 & 2 & 2 & 1 & 4 & 5 & 15,1 \\
\hline 6 & 1 & 5 & 2 & 2 & 1 & 4 & 1 & 3,4 \\
\hline 7 & 5 & 5 & 2 & 4 & 1 & 4 & 5 & 23,9 \\
\hline 8 & 5 & 5 & 2 & 4 & 1 & 4 & 5 & 23,9 \\
\hline 9 & 5 & 5 & 2 & 4 & 1 & 4 & 5 & 23,9 \\
\hline 10 & 5 & 5 & 2 & 4 & 1 & 4 & 5 & 23,9 \\
\hline 11 & 5 & 5 & 2 & 2 & 1 & 4 & 1 & 7,6 \\
\hline 12 & 5 & 5 & 2 & 4 & 1 & 4 & 5 & 23,9 \\
\hline 13 & 5 & 5 & 2 & 3 & 1 & 4 & 1 & 9,3 \\
\hline 14 & 5 & 5 & 2 & 4 & 1 & 4 & 5 & 23,9 \\
\hline 15 & 4 & 5 & 2 & 5 & 1 & 4 & 5 & 23,9 \\
\hline 16 & 4 & 5 & 2 & 5 & 1 & 4 & 5 & 23,9 \\
\hline 17 & 5 & 5 & 2 & 3 & 1 & 3 & 5 & 17,9 \\
\hline 18 & 5 & 5 & 2 & 3 & 1 & 4 & 5 & 20,7 \\
\hline 19 & 5 & 5 & 2 & 3 & 1 & 4 & 5 & 20,7 \\
\hline 20 & 5 & 5 & 2 & 3 & 1 & 4 & 5 & 20,7 \\
\hline 21 & 5 & 5 & 2 & 3 & 1 & 3 & 5 & 17,9 \\
\hline 22 & 5 & 5 & 2 & 3 & 1 & 4 & 5 & 20,7 \\
\hline 23 & 4 & 5 & 2 & 3 & 1 & 4 & 5 & 18,5 \\
\hline 24 & 5 & 5 & 2 & 3 & 1 & 3 & 5 & 17,9 \\
\hline 25 & 5 & 5 & 2 & 3 & 1 & 3 & 5 & 17,9 \\
\hline 26 & 5 & 5 & 2 & 3 & 1 & 4 & 4 & 18,5 \\
\hline 27 & 5 & 5 & 2 & 3 & 1 & 4 & 4 & 18,5 \\
\hline 28 & 5 & 5 & 2 & 3 & 1 & 4 & 4 & 18,5 \\
\hline 29 & 5 & 5 & 2 & 3 & 1 & 4 & 1 & 9,3 \\
\hline 30 & 5 & 5 & 2 & 3 & 1 & 4 & 1 & 9,3 \\
\hline 31 & 5 & 5 & 2 & 3 & 1 & 4 & 2 & 13,1 \\
\hline 32 & 5 & 5 & 2 & 3 & 1 & 4 & 1 & 9,3 \\
\hline 33 & 5 & 5 & 2 & 3 & 1 & 4 & 1 & 9,3 \\
\hline 34 & 5 & 5 & 2 & 3 & 1 & 4 & 4 & 18,5 \\
\hline 35 & 5 & 5 & 2 & 3 & 1 & 4 & 4 & 18,5 \\
\hline 36 & 5 & 5 & 2 & 3 & 1 & 4 & 1 & 18,5 \\
\hline 37 & 5 & 5 & 2 & 3 & 1 & 4 & 1 & 18,5 \\
\hline 38 & 5 & 5 & 2 & 3 & 1 & 4 & 1 & 18,5 \\
\hline $\begin{array}{l}39 \\
\end{array}$ & 5 & 5 & 2 & 3 & 1 & 4 & 1 & 18,5 \\
\hline 40 & 5 & 5 & 2 & 3 & 1 & 4 & 1 & 9,3 \\
\hline 41 & 5 & 5 & 2 & 3 & 1 & 4 & 1 & 17,9 \\
\hline 42 & 3 & 5 & 2 & 3 & 1 & 4 & 1 & 9,3 \\
\hline 43 & 5 & 5 & 2 & 3 & 1 & 4 & 2 & 13,1 \\
\hline 44 & 5 & 5 & 2 & 3 & 1 & 4 & 1 & 13,1 \\
\hline 45 & 5 & 5 & 2 & 3 & 1 & 4 & 4 & 18,5 \\
\hline 46 & 5 & 5 & 2 & 3 & 1 & 4 & 5 & 20,7 \\
\hline 47 & 5 & 5 & 2 & 4 & 1 & 2 & 5 & 16,9 \\
\hline 48 & 5 & 5 & 2 & 3 & 1 & 4 & 5 & 20,7 \\
\hline 49 & 5 & 5 & 2 & 3 & 1 & 4 & 1 & 9,3 \\
\hline 50 & 5 & 5 & 2 & 3 & 1 & 3 & 1 & $\mathbf{8 , 0 0}$ \\
\hline 51 & 5 & 5 & 2 & 3 & 1 & 4 & 1 & 4,1 \\
\hline 52 & 1 & 5 & 2 & 3 & 1 & 4 & 1 & 4,1 \\
\hline
\end{tabular}

Fuente: Elaboración propia. 


\section{Discusión}

La utilización de CVI para el análisis de los puntos débiles de la costa, es una metodología que ha crecido en los últimos años. Una de las ventajas de las que disponen es la facilidad para localizar áreas costeras relativamente concretas, con gran vulnerabilidad a eventos. Algunos autores destacan que los CVI presentan una gran diferencia en la escala de los datos que se utilizan para crear los índices (Koroglu et al., 2019), de manera que permiten simplificar modelizaciones robustas y aplicarlas en áreas relativamente pequeñas.

Existen diferentes modelos de vulnerabilidad en los que se incluyen algunas variables que no aparecen en los modelos originales de (Gornitz, 1994). Para el Mediterráneo resulta de gran utilidad las variables relacionadas con la Posidonia oceanica, una fanerógama marina endémica que, además de suponer un indicador de calidad ambiental de las costas del Mediterráneo, es un agente decisivo contra la erosión costera. Esta variable se ha incluido en índices en la zona de Apulia (Pantusa et al., 2018).

Uno de los problemas de la incorporación de variables en los índices es el aumento de la asimetría (Cogswell et al., 2018; Koroglu et al., 2019). Aunque todas las variables del índice están asociadas a factores que contribuyen a cambios costeros, la contribución de determinadas variables lo hacen en mayor medida que otras (Pendleton et al., 2010), esta sobredimensión puede ser corregida a partir de la ponderación de las variables (Del Río y Gracia, 2009; Gómez-Pazo y Pérez-Alberti, 2017), lo que permite representarlas en función de su importancia. Por otro lado, grandes diferencias en la resolución de los datos, pueden obviar algunas variaciones locales (Pendleton et al., 2010), sobre todo las relacionadas en la variación de la línea de costa.

A pesar de las limitaciones, la integración de los parámetros costeros en un índice permite obtener una visión general de las interacciones físicas y geológicas a lo largo de la costa (Hereher, 2015) y constituyen un primer paso para la toma de decisiones en el litoral, así como para comprender y ayudar a la planificación a largo plazo.

\section{Conclusiones}

La evaluación de la vulnerabilidad costera es crucial para la futura planificación y desarrollo de infraestructuras. La Manga del Mar Menor es un área muy urbanizada en la que el aumento del nivel del mar, por efecto del Cambio Climático, constituye un problema para los servicios básicos actuales.

Las playas de arena y las estructuras artificiales no ofrecen medidas de protección adecuadas contra los factores de estrés climático y están en una mayor exposición a los riesgos, por lo que sería recomendable la adecuación de sistemas duna-playa, que constituyan una barrera para la energía de los temporales que, debido al Cambio Climático, aumentará en los próximos años. 
La adaptación de las playas por medio de regeneraciones artificiales es una medida viable, pero requieren altos esfuerzos económicos a largo plazo o de lo contrario corren el peligro de convertirse en soluciones temporales.

El importante papel de los hábitats naturales, como las praderas marinas y la vegetación dunar proporciona medidas de protección contra los peligros y riesgos costeros. La conservación del hábitat natural y el desarrollo de estrategias de adaptación que se adapten mejor al área local pueden reducir la vulnerabilidad y aumentar la resiliencia de la costa.

El CVI y las herramientas en este estudio ayudan a proporcionar una identificación preliminar para la vulnerabilidad costera de La Manga del Mar Menor, aunque se requieren estudios de sectores de costa más amplios para poder establecer comparativas utilizando metodologías similares y generar información útil en la toma de decisiones y la planificación de la costa.

\section{Referencias}

Adger, W.N. (2006): Vulnerability. Global Environmental Change, 16, 268-281.

Appeaning Addo, K. (2013): Assessing Coastal Vulnerability Index to Climate Change: the Case of Accra -Ghana, Proceedings 12th International Coastal Symposium (Plymouth, England), Journal of Coastal Research, SI 65, 1892-1897.

Baccarin Zanetti, V., Wilson Cabral de Sousa, W. y Débora M. De Freitas, D. (2016): A Climate Change Vulnerability Index and Case Study in a Brazilian Coastal City. Sustainability, 8, 818 .

Ballesteros, G.A. (2014). El turismo de naturaleza en espacios naturales. El caso del Parque Regional de las Salinas y Arenales de San Pedro del Pinatar. Cuadernos de Turismo, 34, 33-51.

Belmonte, F., Romero, M. A., y Ruiz-Sinoga, J.D. (2013): Retroceso de la línea de costa en playas del sur de la Región de Murcia. Scripta Nova, 17, 425- 462.

Bird, E.C.F. (1996): Beach management. Ed Wiley 218 pp.

Church, J.A. y White, N.J. (2011): Sea-level rise from the late 19th to the early 21 st century. Surveys in Geophysics, 32 (4-5): 585-602.

Comisión Europea (2005): Vivir con la erosión costera en Europa: Sedimentos y espacio para la sostenibilidad. Resultados del estudio EUROSION. Pat Doody, M. Ferreira, S. Lombardo, I. Lucius, R. Misdorp, H. Niesing, A. Salman, M. Smallegange, J. Serra Raventós, E. Roca, P. Fernández Bautista, C. Pérez (Eds.). Holanda, 41 p.

Díaz del Río, V. (1990): Estudio ecológico del Mar Menor. Geología (proyecto nº 1005 Medio Marino). Instituto Español de Oceanografía.

Dolan, R., Hayden B.P., May, P. y May S.K. (1980): The reliability of shoreline change measurements from aerial photographs. Shore and Beach, 48(4): 22-29.

Fernández, J.M., Bértola, G.R., Campo, A.M. (2017): Aplicación del Índice de Vulnerabilidad Costera a los barrios costeros del partido de Mar Chiquita, Buenos Aires, Argentina. Geoacta, 42(1): 13-23. 
García-Mora, M.R., Gallego-Fernández, J.B. Williams, A.T. y García-Novo, E. (2001): A coastal dune vulnerability classification. A case study of the SW Iberian Peninsula. Journal of Coastal Research. 17(4):802-811.

Gómez-Pazo, A. y Pérez-Alberti, A. (2017): Vulnerabilidad de las costas de Galicia ante los temporales marinos en el contexto del cambio global. Sémata, Ciencias Sociais e Humanidades, 29, 117-142.

Gornitz, V. Global Coastal Hazards from Future Sea Level Rise (1991): Palaeogeogr. Palaeoclimatol. 89, 379-398.

Gornitz, V. M., \& White, T. W. (1992): A coastal hazards database for the U.S. East coast. ORNL/CDIAC-45, DP-043 A. Oak Ridge National Laboratory, Oak Ridge, Tennessee, U.S. August 1992.

Gornitz, V. M., Daniels, R. C., White, T. W. y Birdwell, K. R. (1994): The development of a coastal risk assessment database: Vulnerability to sea-level rise in the U.S. southeast. Journal of Coastal Research, Special Issue No. 12, p. 327-338.

Hammer-Klose,E.S, Thieler,E.R. (2001): Coastal vulnerability to sea-level rise, a preliminary database for the U.S. Atlantic, Pacific, and Gulf of Mexico coasts.U.S.Geological Survey, Digital Data Series DDS-68, 1CD.

Hernández-Delgado, E. (2015): The emerging threats of climate change on tropical coastal ecosystem services, public health, local economies and livelihood sustainability of small islands: Cumulative impacts and synergies. Marine Pollution Bulletin,101(1), 5-28.

Ibarra Marinas, A.D., Belmonte Serrato, F., Gomariz Castillo, F. y Pérez Cutillas, P. (2015): Evolución de la línea de costa en la Región de Murcia (1956-2013). Geo-Temas, 15, 33-36.

Ibarra Marinas, A.D. (2016): Análisis y evolución de las playas de la Región de Murcia (19562013). Tesis Doctoral. Universidad de Murcia. Murcia.

International Panel on Climate Changes (1990): Climate Change: The IPCC Scientific Assessment; International Panel on Climate Changes (IPC): Cambridge, UK.

International Panel on Climate Changes. Summary for Policy Makers. In Climate Change (2014): Impacts, Adaptation, and Vulnerability. Part A: Global and Sectoral Aspects. Contribution of Working Group II to the Fifth Assessment Report of the Intergovernmental Panel on Climate Change; Field, C.B., Barros, V.R., Dokken, D.J., Mach, K.J., Mastrandrea, M.D., Bilir, T.E., Chatterjee, M., Ebi, K.L., Estrada, Y.O., Genova, R.C., et al., Eds. (2014): International Panel on Climate Changes (IPCC): Cambridge, UK, 2014; pp. $1-32$.

Kantamaneni K., Du X., Aher S., y Singh, R.M. (2017): Building Blocks: A Quantitative Approach for Evaluating Coastal Vulnerability. Water, 9(12): 1-15.

Lillo Carpio, M. (1978): Geomorfología litoral del Mar Menor. Papeles de Geografía, 8, 9-48.

McLaughlin, S. y Cooper, J.A. (2011): A multi-scale coastal vulnerability index: A tool for coastal managers?, Environmental Hazards, 9:3, 233-248

Moore, L.J. and Griggs, G.B. (2002): Long-term cliff retreat and erosion hotspots along the central shores of the Monterey Bay National Marine Sanctuary. Marine Geology 181: 265 283.

Nicholls, R.J , \& Cazenave, A. (2010): Sea-level rise and its impact on coastal zones. Science, 328(5985), 1517-1520. 
Ojeda Zújar, J., Álvarez Francoso, J. I., Martín Cajaraville, D. y Fraile Jurado, P. (2009): El uso de las TIG para el cálculo del indice de vulnerabilidad costera (CVI) ante una potencial subida del nivel del mar en la costa andaluza (España), Geofocus, 9, 83-100.

Overton, M.F.; Grenier, R.R.; Judge, E.K. y Fisher, J.S. (1999): Identification and analysis of coastal erosion and hazard areas: Dare and Brunswick Counties, North Carolina. Journal of Coastal Research, Special Issue, 28, 69-84.

Pilkey, O. H., and Davis, T. W. (1987): An analysis of coastal recession models: North Carolina coast. In: D. Nummedal, O.H. Pilkey and J.D. Howard (Editors), Sea-level Fluctuation and Coastal Evolution. SEPM (Society for Sedimentary Geology) Special Publication No. 41, Tulsa, Oklahoma, p. 59-68.

Rangel-Buitrago N. y Posada-Posada, B.O. (2013): Determinación de la vulnerabilidad y el riesgo costero mediante la aplicación de herramientas SIG y métodos multicriterio. Intropica: Revista del Instituto de Investigaciones Tropicales, 8(1): 29-42.

Riahi, K., Gruebler, A. y Nakicenovic N. (2007): Scenarios of long-term socioeconomic and environmental development under climate stabilization. Technological Forecasting and Social Change, 74(7): 887-935.

Roig i Munar, F.X. (2004): Análisis y consecuencias de la modificación artificial del perfil playa-duna provocado por el efecto mecánico de su limpieza. Investigaciones Geográficas, 33, 87-103.

Roig i Munar, F.X. y Martín Prieto J.A. (2005): Efectos de la retirada de bermas vegetales de la Posidonia oceanica sobre playas de las islas Baleares: consecuencias de la presión turística. Investigaciones Geográficas, 57, 40-52.

Rossoll D., Bermúdez R., Hauss H., Schulz K.G., Riebesell U., Sommer, U., Winder, M. (2012): Ocean Acidification-Induced Food Quality Deterioration Constrains Trophic Transfer. PLoS ONE, 7(4): e34737.

Scott, D., Simpson, M. C., \& Sim, R. (2012): The vulnerability of Caribbean coastal tourism to scenarios of climate change related sea level rise. Journal of Sustainable Tourism,20(6), 883-898.

Shaw, J., Taylor, R.B., Forbes, D.L., Ruz, M.-H., y Solomon, S., (1998): Sensitivity of the Canadian Coast to Sea-Level Rise, Geological Survey of Canada Bulletin 505, 114 p.

Simeoni, U. (2009): Coastal vulnerability related to sea-level rise. Gemorphology, 107(1):1-2.

Thieler, E.R., and Hammar-Klose, E.S. (2000): National assessment of coastal vulnerability to sea-level rise; Preliminary results for the U.S. Atlantic Coast: U.S. Geological Survey Open-File Report, 99-593.

Vidal, C., Losada, M.A., Medina, R. y Losada, L. (1995): Modelos morfodinámicos de las playas. Ingeniería del Agua, 2, 55-74.

Wetzel, F. T., Kissling, W. D., Beissmann, H., \& Penn, D. J. (2012): Future climate change driven sea-level rise: secondary consequences from human displacement for island biodiversity. Global Change Biology, 18(9), 2707-2719. 